\title{
PERIOSTEAL CHONDROMA OF THE FEMUR: A CASE REPORT
}

Ece Şenyiğit ${ }^{1}$, Nur Gülce İşkan ${ }^{1}$, Mert Çiftdemir²

${ }^{1}$ Trakya University School of Medicine, Edirne, TURKEY

${ }^{2}$ Department of Orthopedics and Traumatology, Trakya University School of Medicine, Edirne, TURKEY

\section{ABSTRACT}

Aims: Periosteal chondroma is a rare and benign cartilage tumor seen mostly in long bones and in patients under 30 years of age. In this case report, it is aimed to present a 16-year-old female patient with a periosteal chondroma in the distal femur.

Case Report: A 16-year-old female patient was admitted to the hospital with the history of pain in her distal part of right thigh. A superficial cortical erosion with well-defined borders without any relation to the intramedullary area was seen in magnetic resonance imaging. The lesion was regarded as periosteal chondroma and marginal excision was performed.

Conclusion: This case report shows the importance of the differential diagnosis in cortical lesions. Periosteal chondroma may be considered when a patient has a history of long term pain in adolescent age group.

Keywords: Chondroma, thigh, adolescent, periosteum

\section{INTRODUCTION}

Periosteal chondroma is a rare and benign cartilage tumor. It is located on the surface of the cortical bone (1). It is seen mostly in long bones, particularly the proximal humerus (2). Its predominant region is metaphyses of long bones (3). In addition, it is seen mostly in younger than 30 years of age and the highest frequency is between 10 and 20 years $(1,4)$. Its diagnosis is mainly based on histological characteristics (1).

In this case report, a 16-year-old female patient with a periosteal chondroma in the right distal femur is presented. Written informed consent was obtained from patient's parents.

\section{CASE REPORT}

A 16-year-old female patient was admitted to Orthopedics Department of Trakya University Hospital with an eight-month history of pain in the distal part of her right thigh. The patient described the pain as gradually increasing at night and with exercise. Additionally, she could locate the pain with her finger. In phy- sical examination, the thigh had no edema, erythema nor increase in warmth and the patient had no history of weight loss and limitation of the right knee range of motion. In physical examination, the pathologies which can be associated with these symptoms such as trauma or infection were excluded.

Further analysis was done by plain radiographs and magnetic resonance imaging (MRI). A cortical expansion was seen at the anterior cortex of the distal metaphysis of right femur in plain radiographs. In addition, a small osteolytic lesion at the apex of the expanded anterior femoral cortex with a narrow transition zone was seen. In MRI, there was a superficial erosion with well-defined borders at the apex of the expansion which is $6.4 \mathrm{~mm} \mathrm{X} 4.3 \mathrm{~mm}$ in size and it has no relation with intramedullary area (Figure 1).

The lesion showed hypointense signal properties in $\mathrm{T} 1$ and hyperintense signal properties in T2 weighted sequences. Bone scintigraphy revealed a subcentimetric osteoblastic focus at the anterior cortex of right distal femur. 


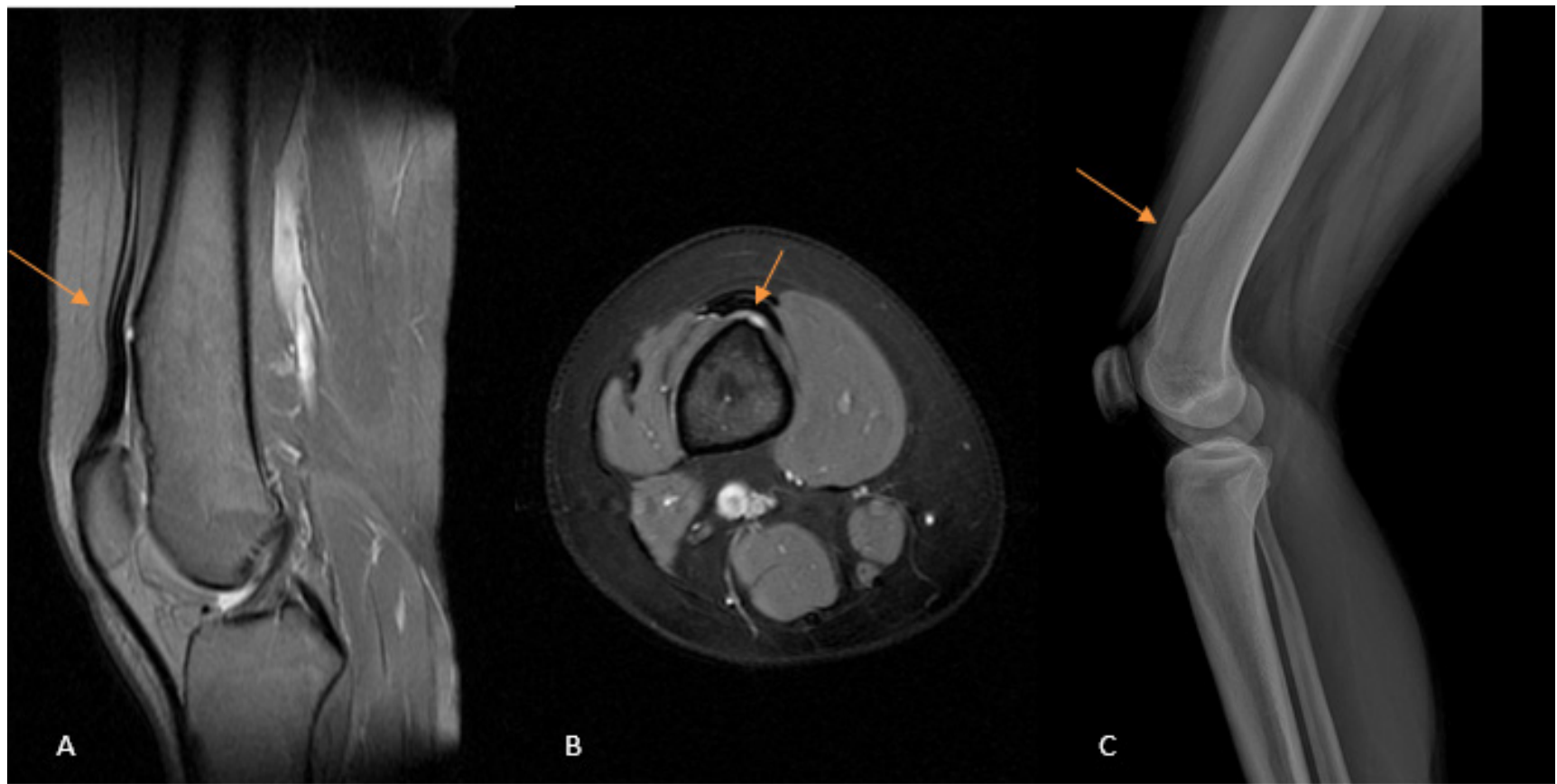

Figure 1: Sagittal and axial T2 Fat-Sat MRI views of right femur (A\&B), Lateral plain radiograph of right femur (C).

Initially, the lesion was regarded as osteoid osteoma. In order to confirm the diagnosis, the patient's response to acetylsalicylic acid was tested, bone scintigraphy and MRI of the patient were examined. The result of acetylsalicylic acid test was negative. According to the test result and absence of a nidus and perilesional bone edema in the MRI, osteoid osteoma was excluded from the differential diagnosis list. The lesion was thought as a periosteal benign lesion such as periosteal chondroma, periosteal ganglion, subperiosteal giant-cell reparative granuloma, superficial aneurysmal bone cyst and surgical treatment had been decided to remove the lesion. In surgery, periosteally located lesion was observed and marginal excision was performed (Figure $2-3)$. Then the material was sent to the pathology department for further examination. Histopathological examination confirmed the diagnosis of periosteal chondroma (Figure 4-5). The patient was seen 3 months after the surgery and she had a full recovery. She had no pain or any other symptoms which were seen before. In MRI, there was no sign of relapse.

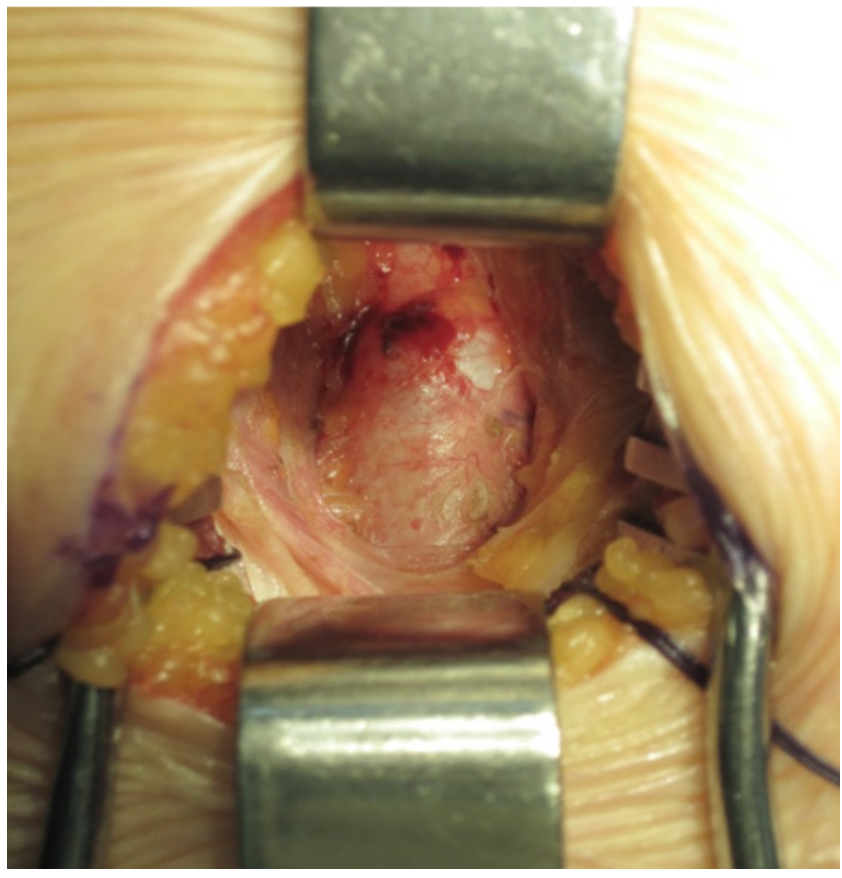

Figure 2: In surgery, the lesion is seen as a hump on the periosteum covering right femur. 


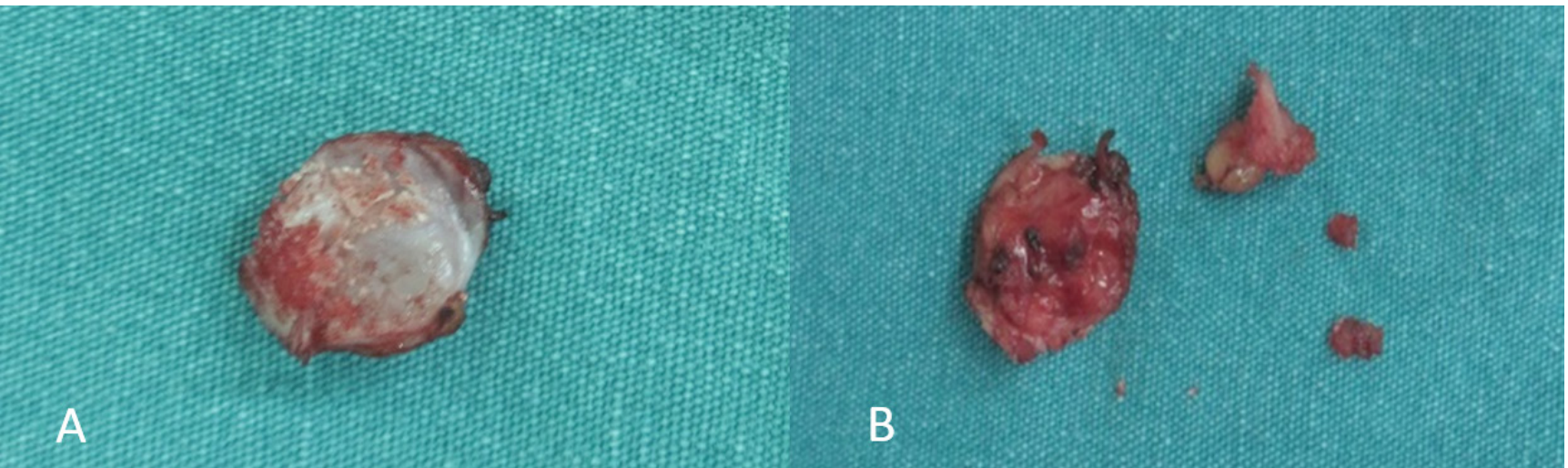

Figure 3: The dorsal aspect of the lesion after the excision (A), the ventral aspect of the lesion covered with periosteum after the excision (B).

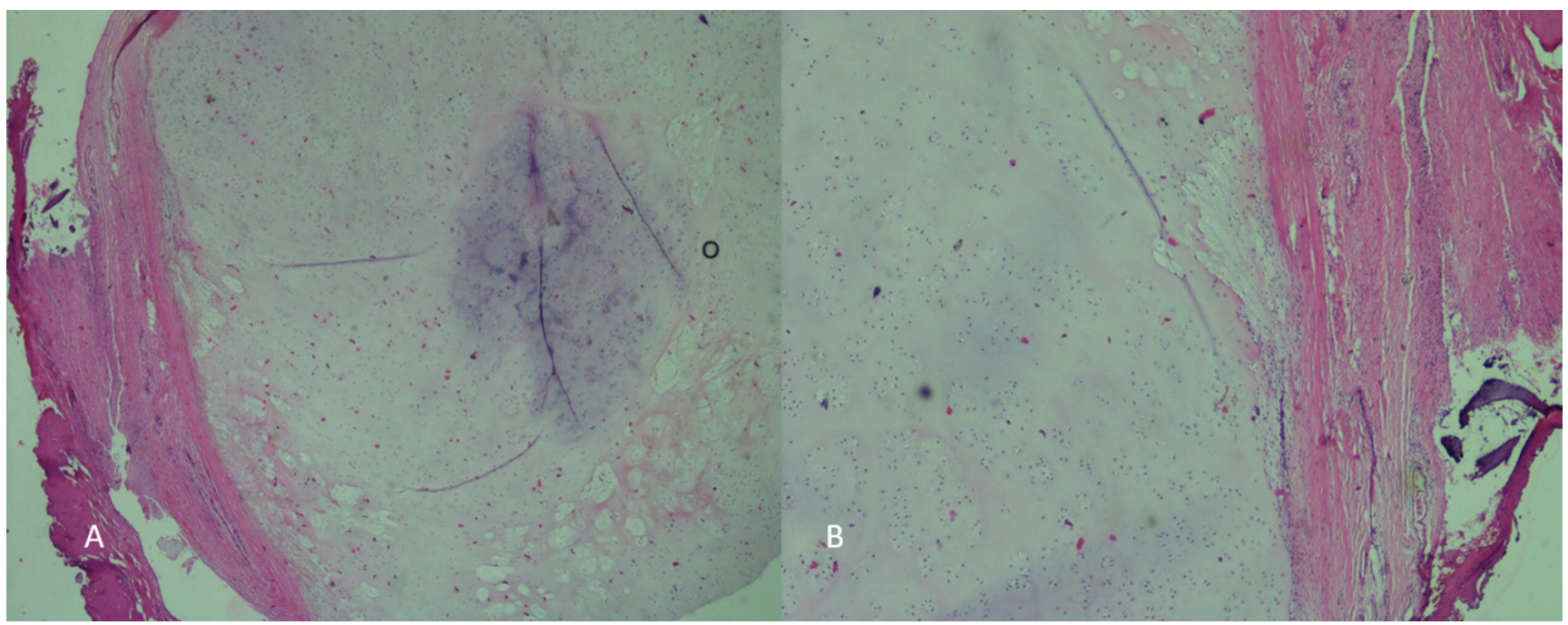

Figure 4: Benign chondroid tumor with a lobulated pattern, adjacent to fibrous periosteal tissue. (A: HEx40, B: HEx100).

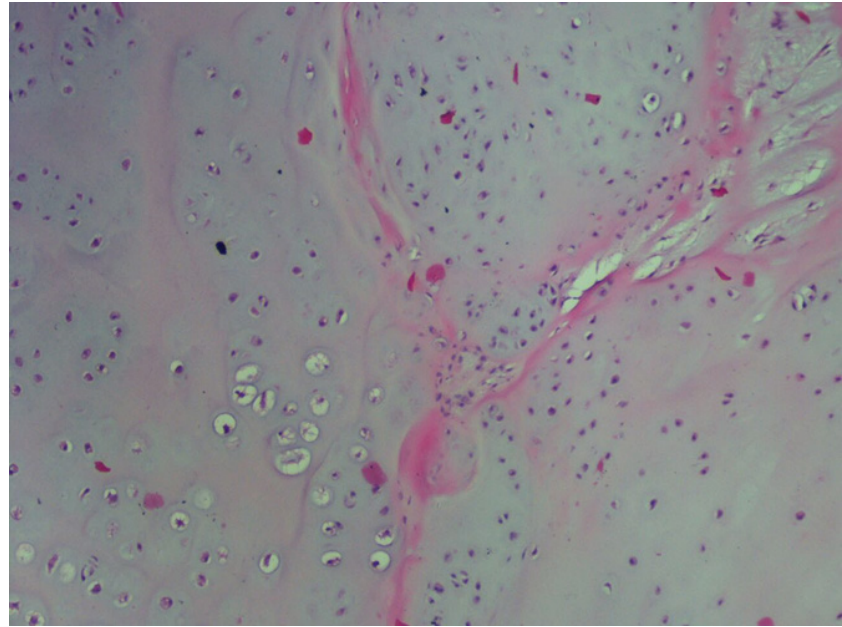

Figure 5: Benign chondroid lobular proliferation with surrounding ossified borders and septation (HEx100).

\section{DISCUSSION}

Periosteal chondroma is a slow-growing lesion of partial size arising within or under the periosteum (5). The lesion is seen mostly in patients younger than 30 -year-old (6). Close collaboration between the surgeon, the radiologist, and the pathologist is needed to achieve the right diagnosis and treatment (4).

Our patient, who was 16 years old, consulted to the hospital with pain in her right knee. Because of the rest pain, three diagnoses were thought: tumor, infections or rheumatologic diseases. However, pain that increases with exercise is not a usual complaint for these diagnoses. Osteoid osteoma is characterized by night-time pain that may be reassured by acetylsalicylic acid or other non-steroid anti-inflammatory drugs $(2,7)$. 
To support the diagnosis of osteoid osteoma, patient's response to acetylsalicylic acid was tested. It was expected to be positive but the result was negative. Osteoid osteoma was excluded from the differential diagnosis list after examining the radiological and clinical features of the lesion and the patient. Eventually, the diagnosis was thought as a benign periosteal lesion and surgical intervention was decided.

In the review and the case report of Kai $\mathrm{Z}$ et al. (1) $50 \%$ of the patients were male, which shows that the distribution of the lesion does not vary between genders. In addition, the mean of age was 14.67 years (1). In this aspect, our case is coherent with the literature.

Estimation of clinical course of periosteal chondroma can help in its diagnosis. Characteristically, the first symptom is localized swelling, followed by more than 6 months of continuous pain (4). In our case, the patient had 8 months history of pain and also night-time pain, but she did not have swelling.

Histopathologically, periosteal chondromas show a protuberant lobular organization of hyaline cartilage and extend from the periosteum toward the adjacent cortical bone. They are usually hypocellular (1). In pathology report of this patient, it is reported that the lesion is cellular and shows focal ossification.

The treatment of periosteal chondroma requires surgical management which consists of en bloc marginal excision and thorough curettage (2). In this case, en bloc marginal excision was preferred.

In conclusion, clinicians should think cortical lesions which are in osteolytic appearance when they face with these kinds of radiological findings as in our patient. Periosteal chondroma is one of them. For the treatment of these kinds of benign lesions, marginal excision provides successful results.

\section{Acknowledgements}

We would like to thank Prof. Ufuk Usta for his contributions.

Ethics Committee Approval: N/A

Informed Consent: Written informed consent was obtained from the patient's parents of this study.

Conflict of Interest: The authors declared no conflict of interest.
Author contributions: Concept: EŞ, NGİ, MÇ. Design: EŞ, NGİ, MÇ. Supervision: EŞ, NGİ, MÇ. Resources: EŞ, NGİ, MÇ. Materials: EŞ, NGİ, MÇ. Data collection and/ or processing: EŞ, NGİ, MÇ. Analysis and/or Interpretation: EŞ, NGİ, MÇ. Literature Search: EŞ, NGİ, MÇ. Writing Manuscript: EŞ, NGİ, MÇ. Critical Review: EŞ, NGİ, MÇ.

Financial disclosure: The authors declared that this study received no financial support.

Editor-in-chief's Note: Two of the authors of this article, Ece Şenyiğit and Nur Gülce İşkan are members of the editorial board of Turkish Medical Student Journal. However, they did not take place in any stage on the editorial decision of the manuscript. The editors who evaluated this manuscript are from another institutions.

\section{REFERENCES}

1. Kai Z, Xiuchun Y, Songfeng X et al. Periosteal chondroma of the femur: a case report and review of the literature. Oncol Lett 2015;9(4):1637-40.

2. Fabbri N, Donati D. Periosteal chondroma. In: Picci P, Manfrini M, Fabbri N et al. Atlas of Musculoskeletal Tumors and Tumorlike Lesions. Switzerland: Springer; 2014.p.57-9.

3. Saraç K, Kaygusuz Akif, Elmalı N. Periosteal chondroma of the hand: two cases. Turgut Özal Tip Merkezi Dergisi 1996;3(1):44-6.

4. Boriani S, Bacchini P, Bertoni F et al. Periosteal chondroma: a review of twenty cases. J Bone Joint Surg Am 1983;65:205-12.

5. Lewis MM, Kenan S, Yabut SM et al. Periosteal chondroma. A report of ten cases and review of the literature. Clin Orthop Relat Res 1990;256:185-92.

6. Akansu B, Atık E, Altintaş S et al. Periosteal chondroma of the ischium; an unusual location. Turk Patoloji Derg 2012;28:172-4.

7. Gökalp MA, Gözen A, Ünsal SŞ et al. An alternative surgical method for treatment of osteoid osteoma. Med Sci Monit 2016;22:580-6. 